\title{
ON INFINITE HORIZON ACTIVE FAULT DIAGNOSIS FOR A CLASS OF NON-LINEAR NON-GAUSSIAN SYSTEMS
}

\author{
IVo PUNČOCHÁř, MiRosLAV ŠIMANDL \\ NTIS-New Technologies for the Information Society, European Centre of Excellence \\ Faculty of Applied Sciences, University of West Bohemia, Univerzitní 8, 306 14, Pilsen, Czech Republic \\ e-mail: \{ivop, simandl\}@kky.zcu.cz
}

\begin{abstract}
The paper considers the problem of active fault diagnosis for discrete-time stochastic systems over an infinite time horizon. It is assumed that the switching between a fault-free and finitely many faulty conditions can be modelled by a finite-state Markov chain and the continuous dynamics of the observed system can be described for the fault-free and each faulty condition by non-linear non-Gaussian models with a fully observed continuous state. The design of an optimal active fault detector that generates decisions and inputs improving the quality of detection is formulated as a dynamic optimization problem. As the optimal solution obtained by dynamic programming requires solving the Bellman functional equation, approximate techniques are employed to obtain a suboptimal active fault detector.
\end{abstract}

Keywords: active fault detection, non-linear stochastic systems, optimal input design, dynamic programming.

\section{Introduction}

The detection and diagnosis of abrupt faults in the dynamics of a system play a vital role in many applications, as timely and reliable decisions help to reduce financial costs and avoid hazardous situations (Isermann, 2006).

Most approaches to fault detector design can be called passive because they lead to fault detectors that passively process observed data and generate decisions (Basseville and Nikiforov, 1993; Puig, 2010). Although passive fault detectors deliver a satisfactory quality of detection in many cases, they may fail to provide timely and reliable decisions when the observed data are not informative enough (Campbell and Nikoukhah, 2004). To deal with such situations, gradual use of ideas from optimum experimental designs (Kiefer, 1959; Atkinson and Donev, 1992), parameter estimation (Mehra, 1974; Goodwin and Payne, 1977), and control (Nett, 1986) in the area of fault detection has resulted in an active approach to fault detector design.

An active fault detector generates a decision and an input signal that is injected into the system in order to improve the quality of detection. Such a problem was addressed first for stochastic models (Zhang, 1989; Kerestecioğlu, 1993) and later on for deterministic uncertain models (Scola et al., 2003; Campbell and Nikoukhah, 2004; Andjelkovic et al., 2008; Niemann, 2012; Ashari et al., 2012a; Scott et al., 2013). The input signal designed to improve the quality of detection can be inconvenient from a control perspective. Therefore, integrated active fault detection and control for deterministic uncertain models (Niemann, 2006; Poulsen and Niemann, 2008; Ashari et al., 2012b) and stochastic models (Blackmore et al., 2008) was studied.

A unified formulation of active fault detection and control for stochastic systems over a finite time horizon was introduced by Šimandl and Punčochář (2009). As the optimal solution based on the closed-loop information processing strategy (Bar-Shalom, 1981) poses a complex functional problem, approximation techniques were used to obtain suboptimal solutions for several special cases of the unified formulation (Šimandl et al., 2005; Široký et al., 2011). However, these approximation techniques are suitable only for linear systems or suffer from substantial on-line computational demands.

Another point is that active fault detection is usually considered over a finite test period during which a specially designed input signal is injected into the system to reveal a potential fault (Campbell and Nikoukhah, 2004; Šimandl et al., 2005; Blackmore et al., 2008). However, if the system is operated continuously, the length of the test period and the time when the test period 
starts need to be determined using a heuristic rule or through another optimization. One way to completely circumvent this issue is to perform active fault detection continuously while ensuring that it does not have a detrimental impact on control or other aims.

This paper presents active fault diagnosis for a class of non-linear non-Gaussian systems that allow active fault diagnosis to be performed continuously over an infinite time horizon. The goal is to formulate the design of the active fault detector as a dynamic optimization problem over an infinite time horizon with a discounted criterion and provide a solution procedure based on approximate dynamic programming.

The paper is organized as follows. The active fault detection problem over an infinite time horizon is formulated in Section 2 The design of the approximate active fault detector is discussed in Section 3 . First, the original problem is reformulated in Section 3.1 using a hyper-state as a perfect state information problem. Then, the optimal solution based on solving the Bellman functional equation is provided in Section 3.2 and the approximate solution is discussed in Section 3.3 . The proposed approximate active fault detector is demonstrated in a numerical example in Section 4. Concluding remarks are given in Section 5

\section{Problem formulation}

The block diagram of active fault detection and diagnosis is depicted in Fig. 1. It consists of a given system and an active fault detector that are described in the following subsections.

2.1. System. The problem of active fault detection is considered on an infinite time horizon for a system that can be described at each time step $k \in \mathcal{T}=\{0,1,2, \ldots\}$ by the following discrete-time non-linear model:

$$
\mathbf{x}_{k+1}=\mathbf{f}\left(\mathbf{x}_{k}, \mu_{k}, \mathbf{u}_{k}\right)+\mathbf{w}_{k},
$$

where $\mathbf{x}_{k} \in \mathbb{R}^{n_{x}}$ is a known continuous-valued vector and $\mu_{k} \in \mathcal{M}=\{1,2, \ldots, N\}$ is an unknown discrete-valued scalar. Taken together, $\mathbf{x}_{k}$ and $\mu_{k}$ represent the hybrid state $\mathbf{x}_{k}^{\mathrm{a}}=\left[\mathbf{x}_{k}^{\mathrm{T}}, \mu_{k}\right]^{\mathrm{T}} \in \mathcal{X} \triangleq$ $\mathbb{R}^{n_{x}} \times \mathcal{M}$ of the system. The input $\mathbf{u}_{k} \in \mathcal{U} \subset \mathbb{R}^{n_{u}}$ can attain only values from a discrete set of admissible inputs $\mathcal{U}=\left\{\overline{\mathbf{u}}^{1}, \overline{\mathbf{u}}^{2}, \ldots, \overline{\mathbf{u}}^{M}\right\}$. The additive white state

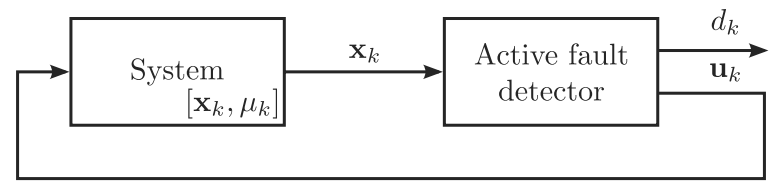

Fig. 1. Block diagram of active fault detection and diagnosis. noise $\mathbf{w}_{k} \in \mathbb{R}^{n_{x}}$ has a known conditional probability density function (pdf) $p_{\mathbf{w}}\left(\mathbf{w}_{k} \mid \mathbf{x}_{k}^{\mathrm{a}}\right)$. It is assumed that the state noise $\mathbf{w}_{k}$ has the whole $\mathbb{R}^{n_{x}}$ as its support, and the conditional mean value $\mathbf{m}_{\mathbf{w}}\left(\mathbf{x}_{k}^{\mathrm{a}}\right)$ and covariance matrix $\mathbf{P}_{\mathbf{w}}\left(\mathbf{x}_{k}^{\mathrm{a}}\right)$ are well defined for all hybrid states $\mathbf{x}_{k}^{\mathrm{a}} \in$ $\mathcal{X}$. The variable $\mu_{k}$ is the index of the model in effect during the $k$-th sampling period, and the known non-linear vector function $\mathrm{f}: \mathcal{X} \times \mathcal{U} \mapsto \mathbb{R}^{n_{x}}$ has the following structure:

$$
\mathbf{f}\left(\mathbf{x}_{k}, \mu_{k}, \mathbf{u}_{k}\right)= \begin{cases}\mathbf{f}_{1}\left(\mathbf{x}_{k}, \mathbf{u}_{k}\right) & \text { if } \mu_{k}=1, \\ \mathbf{f}_{2}\left(\mathbf{x}_{k}, \mathbf{u}_{k}\right) & \text { if } \mu_{k}=2, \\ \vdots & \\ \mathbf{f}_{N}\left(\mathbf{x}_{k}, \mathbf{u}_{k}\right) & \text { if } \mu_{k}=N,\end{cases}
$$

where $\mathbf{f}_{i}: \mathbb{R}^{n_{x}} \times \mathcal{U} \mapsto \mathbb{R}^{n_{x}}$ is a given non-linear function that represents the behaviour of the system under the fault-free condition for $i=1$ and under faulty conditions for $1<i \leq N$. The switching between fault-free and faulty conditions is assumed to be random and described by a stationary finite-state Markov chain with known transition probabilities $P_{i, j}=P\left(\mu_{k+1}=j \mid \mu_{k}=i\right)$. Finally, the initial conditions $\mathbf{x}_{0}$ and $\mu_{0}$ are mutually independent and have a given pdf $p\left(\mathbf{x}_{0}\right)$ and a discrete probability distribution $P\left(\mu_{0}\right)$, respectively.

2.2. Active fault detector. The active fault detector that generates decisions about faults and inputs can generally be described by a causal relation of the form

$$
\left[\begin{array}{l}
d_{k} \\
\mathbf{u}_{k}
\end{array}\right]=\boldsymbol{\rho}_{k}\left(\mathbf{I}_{0}^{k}\right)
$$

where $\mathbf{I}_{0}^{k}=\left[\mathbf{x}_{0}^{k}, \mathbf{u}_{0}^{k-1}\right]$ is an information vector containing the data received up to the time step $k$ and $\boldsymbol{\rho}_{k}$ : $\mathbb{R}^{n_{x}(k+1)} \times \mathcal{U}^{k} \mapsto \mathcal{M} \times \mathcal{U}$ is an unknown non-linear vector function that represents the active fault detector. Since the decision $d_{k} \in \mathcal{M}$ can be interpreted as a point estimate of $\mu_{k}$, it provides both fault detection and fault diagnosis information. The notation $\mathbf{x}_{i}^{j}=\left[\mathbf{x}_{i}^{\mathrm{T}}, \mathbf{x}_{i+1}^{\mathrm{T}}, \ldots, \mathbf{x}_{j}^{\mathrm{T}}\right]^{\mathrm{T}}$ is used to denote a sequence of variables from the time step $i$ through the time step $j$. The sequence $\rho^{\mathrm{p}}=$ $\left\{\boldsymbol{\rho}_{0}, \boldsymbol{\rho}_{1}, \ldots\right\}$ of functions $\boldsymbol{\rho}_{k}$ is called the policy and needs to be determined such that a chosen design criterion representing the performance of the active fault detector is optimized.

2.3. Design criterion. The performance of the active fault detector is assessed using the following additive discounted criterion:

$$
J\left(\boldsymbol{\rho}^{\mathrm{p}}\right)=\lim _{F \rightarrow \infty} \mathrm{E}\left\{\sum_{k=0}^{F} \lambda^{k} L^{\mathrm{d}}\left(\mu_{k}, d_{k}\right)\right\},
$$


where $\lambda \in(0,1)$ is a discount factor per sampling period, $L^{\mathrm{d}}: \mathcal{M} \times \mathcal{M} \mapsto \mathbb{R}^{+}$is a detection cost function that penalizes the discrepancy between the decision $d_{k}$ and the actual model $\mu_{k}, \mathbb{R}^{+}$denotes the set of non-negative real numbers, and $\mathrm{E}\{\cdot\}$ is the expectation operator over all included random variables.

To ensure that the criterion (4) is finite for every policy $\rho^{\mathrm{p}}$, the detection cost function $L^{\mathrm{d}}$ is assumed to be bounded,

$$
L_{\min }^{\mathrm{d}} \leq L^{\mathrm{d}}\left(\mu_{k}, d_{k}\right) \leq L_{\max }^{\mathrm{d}},
$$

where $L_{\min }^{\mathrm{d}}=\min _{\mu_{k}, d_{k}} L^{\mathrm{d}}\left(\mu_{k}, d_{k}\right) \geq 0$ and $L_{\max }^{\mathrm{d}}=$ $\max _{\mu_{k}, d_{k}} L^{\mathrm{d}}\left(\mu_{k}, d_{k}\right)<\infty$ are finite lower and upper bounds on the detection cost function, respectively. Moreover, for the design criterion to be meaningful, the detection cost function should penalize the correct decision less than incorrect ones. Thus, it is assumed that for each $\mu_{k} \in \mathcal{M}$ the detection cost function satisfies

$$
L^{\mathrm{d}}\left(\mu_{k}, \mu_{k}\right)<L^{\mathrm{d}}\left(\mu_{k}, d_{k}\right), \quad d_{k} \in \mathcal{M}, \quad d_{k} \neq \mu_{k} .
$$

This means that for a given $\mu_{k} \in \mathcal{M}$ the detection cost function $L^{\mathrm{d}}$ attains a unique minimum at $d_{k}=\mu_{k}$. Note that for a given decision $d_{k}$ the minimum of the detection cost function does not need to be at $\mu_{k}=d_{k}$ as there is neither theoretical nor practical reason for making such an assumption. Using the lower and upper bounds on the detection cost function, it is possible to derive the lower and upper bounds on the value of the criterion (4) under any admissible policy $\rho^{\mathrm{p}}$ as

$$
\frac{L_{\min }^{\mathrm{d}}}{1-\lambda} \leq J\left(\boldsymbol{\rho}^{\mathrm{p}}\right) \leq \frac{L_{\max }^{\mathrm{d}}}{1-\lambda}
$$

The goal is to determine the optimal policy $\rho^{\mathrm{p} *}$ that minimizes the criterion (4),

$$
J^{*}=J\left(\boldsymbol{\rho}^{\mathrm{p} *}\right)=\inf _{\boldsymbol{\rho}^{p}} J\left(\boldsymbol{\rho}^{\mathrm{p}}\right) .
$$

The discount factor $\lambda$ can be interpreted in two different ways. First, it can be understood as a tuning parameter that makes the criterion well defined and allows more emphasis to be put on immediate decisions. Another interpretation that is common in investment problems (Denardo, 2003) can be provided, when the detection cost function $L^{\mathrm{d}}\left(\mu_{k}, d_{k}\right)$ represents financial costs connected with fault detection (costs of maintenance, missed detection, false alarm, etc.). Assuming that money spent during fault detection could otherwise be invested with a per sampling period interest rate $r>0$, the overall expected costs of the fault detection over a finite time horizon $F$ can be expressed as

$$
\bar{J}(F)=\mathrm{E}\left\{\sum_{k=0}^{F}(1+r)^{F-k} L^{\mathrm{d}}\left(\mu_{k}, d_{k}\right)\right\} .
$$

Since the interest rate $r$ is positive, the overall expected costs would approach infinity for the infinite horizon $F$. Nevertheless, by factoring out the term $(1+r)^{F}$, the detection costs can be rewritten as

$$
\bar{J}(F)=(1+r)^{F} \mathrm{E}\left\{\sum_{k=0}^{F} \lambda^{k} L^{\mathrm{d}}\left(\mu_{k}, d_{k}\right)\right\},
$$

where $\lambda=1 /(1+r)$. Therefore, the criterion

$$
J\left(\boldsymbol{\rho}^{\mathrm{p}}\right)=\lim _{F \rightarrow \infty} \frac{\bar{J}(F)}{(1+r)^{F}}
$$

can be regarded as the overall costs of fault detection expressed at time step 0 .

\section{Design of an approximate active fault detector}

The design of the active fault detector as formulated in the previous section is a dynamic optimization problem that does not permit analytical solution and is computationally intractable. Therefore, an approximate active fault detector is designed in the following three steps:

Step 1. The original problem formulation falls into the class of imperfect state information problems (Bertsekas, 1995). In order to use a standard form of dynamic programming, the original problem is first reformulated as a perfect state information one by introducing a new state, called the hyper-state or the information state (Åström, 1965).

Step 2. The optimal active fault detector is derived as a solution to the Bellman functional equation that corresponds to the reformulated problem obtained in the previous step.

Step 3. The approximate active fault detector is obtained by solving the Bellman functional equation numerically. The numerical solution uses the piecewise constant approximation of the value function, and the value function iteration algorithm is performed over sample points in the hyper-state space.

3.1. Problem reformulation. The original problem presented in Section 2 is reformulated as a perfect state information problem by introducing a directly observed hyper-state $\mathbf{s}_{k}$ that is a function of the information vector $\mathbf{I}_{0}^{k}$ and represents a sufficient statistic for the problem considered. As the continuous-valued variable $\mathbf{x}_{k}$ is already observed directly, only a sufficient statistic for $\mu_{k}$ is required. One of the possible sufficient statistics is represented by the conditional 
probability distribution $P\left(\mu_{k} \mid \mathbf{I}_{0}^{k}\right)$ denoted as the belief state (Bertsekas, 1995; Åström, 1965). As the conditional probabilities add up to one, the belief state reduced by one dimension is used,

$$
\mathbf{b}_{k}=\left[\begin{array}{c}
b_{k, 1} \\
b_{k, 2} \\
\vdots \\
b_{k, N-1}
\end{array}\right]=\left[\begin{array}{c}
P\left(\mu_{k}=1 \mid \mathbf{x}_{0}^{k}, \mathbf{u}_{0}^{k-1}\right) \\
P\left(\mu_{k}=2 \mid \mathbf{x}_{0}^{k}, \mathbf{u}_{0}^{k-1}\right) \\
\vdots \\
P\left(\mu_{k}=N-1 \mid \mathbf{x}_{0}^{k}, \mathbf{u}_{0}^{k-1}\right)
\end{array}\right],
$$

where $\mathbf{b}_{k} \in \mathcal{B}=\left\{\mathbf{b} \in \mathbb{R}^{N-1}: \mathbf{b} \geq \mathbf{0}, \mathbf{1}^{\mathrm{T}} \mathbf{b} \leq 1\right\}$ is the vector of conditional probabilities, $\mathbf{0}^{\mathrm{T}}=[0,0, \ldots, 0] \in$ $\mathbb{R}^{N-1}$ is the zero vector, $\mathbf{1}^{\mathrm{T}}=[1,1, \ldots, 1] \in \mathbb{R}^{N-1}$ denotes the vector of ones, and the inequalities are component-wise.

The hyper-state $\mathbf{s}_{k}$ consists of $\mathbf{x}_{k}$ and $\mathbf{b}_{k}$,

$$
\mathbf{s}_{k}=\left[\begin{array}{l}
\mathbf{x}_{k} \\
\mathbf{b}_{k}
\end{array}\right] \in \mathcal{S}=\left(\mathbb{R}^{n_{x}} \times \mathcal{B}\right) \subset \mathbb{R}^{n_{s}},
$$

and the original model (1) can be replaced by a new model,

$$
\mathbf{s}_{k+1}=\phi\left(\mathbf{s}_{k}, \mathbf{u}_{k}, \mathbf{x}_{k+1}\right),
$$

where $\phi: \mathcal{S} \times \mathcal{U} \times \mathbb{R}^{n_{x}} \mapsto \mathcal{S}$ is a vector non-linear function and $\mathbf{x}_{k+1}$ can be regarded as a disturbance with the conditional pdf $p\left(\mathbf{x}_{k+1} \mid \mathbf{s}_{k}, \mathbf{u}_{k}\right)=p\left(\mathbf{x}_{k+1} \mid \mathbf{x}_{0}^{k}, \mathbf{u}_{0}^{k}\right)$.

It follows from the definition of the hyper-state that the non-linear function $\phi$ has the following structure:

$$
\phi\left(\mathbf{s}_{k}, \mathbf{u}_{k}, \mathbf{x}_{k+1}\right)=\left[\begin{array}{c}
\mathbf{x}_{k+1} \\
\phi_{1}^{\mathrm{b}}\left(\mathbf{s}_{k}, \mathbf{u}_{k}, \mathbf{x}_{k+1}\right) \\
\phi_{2}^{\mathrm{b}}\left(\mathbf{s}_{k}, \mathbf{u}_{k}, \mathbf{x}_{k+1}\right) \\
\vdots \\
\phi_{N-1}^{\mathrm{b}}\left(\mathbf{s}_{k}, \mathbf{u}_{k}, \mathbf{x}_{k+1}\right)
\end{array}\right]
$$

where $\phi_{i}^{\mathrm{b}}: \mathcal{S} \times \mathcal{U} \times \mathbb{R}^{n_{x}} \mapsto[0,1]$ is a function that represents the optimal recursive estimator of the conditional probability of the $i$-th model. These functions are given by the following recursive relations:

$$
\begin{aligned}
\phi_{i}^{\mathrm{b}}\left(\mathbf{s}_{k}, \mathbf{u}_{k}, \mathbf{x}_{k+1}\right)= & \sum_{j=1}^{N} P\left(\mu_{k+1}=i \mid \mu_{k}=j\right) \\
& \times P\left(\mu_{k}=j \mid \mathbf{x}_{0}^{k+1}, \mathbf{u}_{0}^{k}\right),
\end{aligned}
$$

where the conditional probability $P\left(\mu_{k}=j \mid \mathbf{x}_{0}^{k+1}, \mathbf{u}_{0}^{k}\right)$ is computed as

$$
\begin{aligned}
& P\left(\mu_{k}=j \mid \mathbf{x}_{0}^{k+1}, \mathbf{u}_{0}^{k}\right) \\
& \quad=\frac{p\left(\mathbf{x}_{k+1} \mid \mathbf{x}_{0}^{k}, \mathbf{u}_{0}^{k}, \mu_{k}=j\right) P\left(\mu_{k}=j \mid \mathbf{x}_{0}^{k}, \mathbf{u}_{0}^{k-1}\right)}{p\left(\mathbf{x}_{k+1} \mid \mathbf{x}_{0}^{k}, \mathbf{u}_{0}^{k}\right)} .
\end{aligned}
$$

The conditional pdf $p\left(\mathbf{x}_{k+1} \mid \mathbf{x}_{0}^{k}, \mathbf{u}_{0}^{k}\right)$ is given as

$$
\begin{aligned}
p\left(\mathbf{x}_{k+1} \mid \mathbf{x}_{0}^{k}, \mathbf{u}_{0}^{k}\right)= & \sum_{i=1}^{N} p\left(\mathbf{x}_{k+1} \mid \mathbf{x}_{0}^{k}, \mathbf{u}_{0}^{k}, \mu_{k}=i\right) \\
& \times P\left(\mu_{k}=i \mid \mathbf{x}_{0}^{k}, \mathbf{u}_{0}^{k-1}\right),
\end{aligned}
$$

and the likelihood function $p\left(\mathbf{x}_{k+1} \mid \mathbf{x}_{0}^{k}, \mathbf{u}_{0}^{k}, \mu_{k}=j\right)$ is

$$
\begin{aligned}
& p\left(\mathbf{x}_{k+1} \mid \mathbf{x}_{0}^{k}, \mathbf{u}_{0}^{k}, \mu_{k}=j\right) \\
& \quad=p_{\mathbf{w}}\left(\mathbf{x}_{k+1}-\mathbf{f}_{j}\left(\mathbf{x}_{k}, \mathbf{u}_{k}\right) \mid \mathbf{x}_{k}, \mu_{k}=j\right) .
\end{aligned}
$$

Finally, the initial state $\mathbf{s}_{0}$ is given by the directly observed $\mathbf{x}_{0}$ and the probabilities $P\left(\mu_{0}=j\right)$ for $j=$ $1, \ldots, N-1$. The obtained model is a perfect state information model because the hyper-state $\mathbf{s}_{k}$ is known at each time step $k$.

As the hyper-state $\mathbf{s}_{k}$ is a sufficient statistic, which summarizes all relevant information contained in the information vector $\mathbf{I}_{0}^{k}$, the active fault detector (3) and the design criterion (4) can be updated as follows. Instead of the time-varying active fault detector (3), it is sufficient to consider only a stationary active fault detector of the form

$$
\left[\begin{array}{l}
d_{k} \\
\mathbf{u}_{k}
\end{array}\right]=\boldsymbol{\rho}\left(\mathbf{s}_{k}\right)
$$

where $\rho: \mathcal{S} \mapsto \mathcal{M} \times \mathcal{U}$ is an unknown stationary policy. Using a similar reasoning, the original design criterion (4) can be replaced by the following equivalent criterion:

$$
\bar{J}\left(\boldsymbol{\rho}, \mathbf{s}_{0}\right)=\lim _{F \rightarrow \infty} \mathrm{E}\left\{\sum_{k=0}^{F} \lambda^{k} \bar{L}^{\mathrm{d}}\left(\mathbf{s}_{k}, d_{k}\right) \mid \mathbf{s}_{0}\right\},
$$

where $\mathrm{E}\{\cdot \mid \cdot\}$ represents the conditional expectation operator and $\bar{L}^{\mathrm{d}}: \mathcal{S} \times \mathcal{M} \mapsto \mathbb{R}^{+}$is an equivalent detection cost function derived from the detection cost function $L^{\mathrm{d}}\left(\mu_{k}, d_{k}\right)$ as

$$
\bar{L}^{\mathrm{d}}\left(\mathbf{s}_{k}, d_{k}\right)=\mathrm{E}\left\{L^{\mathrm{d}}\left(\mu_{k}, d_{k}\right) \mid \mathbf{x}_{0}^{k}, \mathbf{u}_{0}^{k-1}, d_{k}\right\} .
$$

As the conditional probabilities $P\left(\mu_{k}=j \mid \mathbf{x}_{0}^{k}, \mathbf{u}_{0}^{k-1}, d_{k}\right)$ are the belief states contained in the hyper-state $\mathbf{s}_{k}$, the equivalent detection cost function is easy to evaluate for a given hyper-state and decision.

A relation to the original design criterion (4) can be established in the following way. By substituting (22) into (21) and applying the chain rule, 21) can be rewritten as

$$
\bar{J}\left(\boldsymbol{\rho}, \mathbf{s}_{0}\right)=\lim _{F \rightarrow \infty} \mathrm{E}\left\{\sum_{k=0}^{F} \lambda^{k} L^{\mathrm{d}}\left(\mu_{k}, d_{k}\right) \mid \mathbf{s}_{0}\right\} .
$$

Using the Weierstrass theorem, it can be shown that the series in 23) converges uniformly on $\mathcal{S}$. Thus, the 
interchange of the expectation and limit operators can be done and provides the following relation:

$$
\mathrm{E}\left\{\bar{J}\left(\boldsymbol{\rho}, \mathbf{s}_{0}\right)\right\}=J(\boldsymbol{\rho}) .
$$

Hence, the optimal active fault detector that minimizes the equivalent criterion (21) for every $\mathbf{s}_{0} \in \mathcal{S}$ also minimizes the original criterion (4). For more details on problem reformulation using sufficient statistics, see, e.g., the work of Bertsekas (1995).

3.2. Optimal active fault detector. The design of an optimal active fault detector is based on solving a functional equation (Puterman, 2005; Buşoniu et al., 2010). Let us define a value function $V^{\boldsymbol{\rho}}\left(\mathbf{s}_{k}\right)$ that represents the value of the design criterion (21) when starting at the state $\mathbf{s}_{k}$ and following the stationary policy $\boldsymbol{\rho}$. The optimal value function $V^{*}\left(\mathbf{s}_{k}\right)=V^{\boldsymbol{\rho}^{*}}\left(\mathbf{s}_{k}\right)$ has to satisfy the following Bellman functional equation for each state $\mathbf{s}_{k} \in \mathcal{S}$ :

$$
\begin{aligned}
& V^{*}\left(\mathbf{s}_{k}\right) \\
& =\min _{\substack{d_{k} \in \mathcal{M} \\
\mathbf{u}_{k} \in \mathcal{U}}} \mathrm{E}\left\{\bar{L}^{\mathrm{d}}\left(\mathbf{s}_{k}, d_{k}\right)+\lambda V^{*}\left(\mathbf{s}_{k+1}\right) \mid \mathbf{s}_{k}, \mathbf{u}_{k}, d_{k}\right\},
\end{aligned}
$$

where $V^{*}: \mathcal{S} \mapsto \mathbb{R}$ is the optimal value function. As the equivalent detection cost function $\bar{L}^{\text {d }}$ does not depend on the input $\mathbf{u}_{k}$ and the conditional expectation of the optimal value function $V^{*}$ does not depend on the decision $d_{k}$, the functional equation (25) can be rewritten as

$$
\begin{aligned}
V^{*}\left(\mathbf{s}_{k}\right)= & \min _{d_{k} \in \mathcal{M}} \mathrm{E}\left\{\bar{L}^{\mathrm{d}}\left(\mathbf{s}_{k}, d_{k}\right) \mid \mathbf{s}_{k}, d_{k}\right\} \\
& +\lambda \min _{\mathbf{u}_{k} \in \mathcal{U}} \mathrm{E}\left\{V^{*}\left(\mathbf{s}_{k+1}\right) \mid \mathbf{s}_{k}, \mathbf{u}_{k}\right\} .
\end{aligned}
$$

This form of the functional equation shows that the optimal decision $d_{k}^{*}$ and the optimal input signal $\mathbf{u}_{k}^{*}$ are given as

$$
\begin{aligned}
& d_{k}^{*}=\sigma^{*}\left(\mathbf{s}_{k}\right)=\arg \min _{d_{k} \in \mathcal{M}} \bar{L}^{\mathrm{d}}\left(\mathbf{s}_{k}, d_{k}\right), \\
& \mathbf{u}_{k}^{*}=\gamma^{*}\left(\mathbf{s}_{k}\right)=\arg \min _{\mathbf{u}_{k} \in \mathcal{U}} \mathrm{E}\left\{V^{*}\left(\mathbf{s}_{k+1}\right) \mid \mathbf{s}_{k}, \mathbf{u}_{k}\right\},
\end{aligned}
$$

where $\sigma^{*}: \mathcal{S} \mapsto \mathcal{M}$ and $\gamma^{*}: \mathcal{S} \mapsto \mathcal{U}$ are parts of the optimal stationary policy $\rho^{*}$ that describe the optimal fault detector and the optimal input signal generator, respectively.

It is obvious from 27 that the optimal decision $d_{k}^{*}$ minimizes only the immediate expected detection cost resulting from that particular decision. Therefore, the optimal fault detector can be obtained without solving the functional equation (26). On the other hand, the optimal input $\mathbf{u}_{k}^{*}$ minimizes the discounted sum of the future expected detection costs incurred from the time step $k+1$ through infinity encoded in the optimal value function $V^{*}$.
Since the functional equation (26) is independent of the actual measurements, it can be advantageously solved off-line to obtain the optimal value function $V^{*}$ and thus, in turn, the optimal input signal generator. The optimal active fault detector represented by (27) and (28) can be used on-line to generate the optimal decision and input signal based on the hyper-state $\mathbf{s}_{k}$ that is recursively computed on-line as well.

To compute the optimal input signal from the optimal value function in (28), the conditional expectation $\mathrm{E}\left\{V^{*}\left(\mathbf{s}_{k+1}\right) \mid \mathbf{s}_{k}, \mathbf{u}_{k}\right\}$ needs to be evaluated on-line. In order to reduce on-line computation requirements, the conditional expectation can be evaluated off-line. Then the on-line computation of the optimal input signal consists in a simple enumerative optimization problem over all admissible inputs.

3.3. Approximate active fault detector. Note that the functional equation (26) is highly non-linear due to the minimization operator over all admissible input values and does not permit an analytical solution. A numerical solution relies on the value iteration, policy iteration, or the policy search algorithm (Buşoniu et al., 2010; Powell, 2007; Bertsekas, 1995). In this paper, the value iteration algorithm and a finite-dimensional approximation of the value function are used to find an approximate solution to the functional equation numerically. To this end, sample points in the hyper-state space are chosen, and the value function is approximated on a relevant part of the hyper-state space using a piecewise constant function approximator (Buşoniu et al., 2010). The sample points can be chosen based on the a-priori information to form a grid in the hyper-state space (Denardo, 2003), or they are chosen rather randomly by simulating the system under conditions that are believed to be representative (Powell, 2007; Lee et al., 2006).

3.3.1. Grid and aggregation function design. Grid design techniques originally proposed for the point-mass estimation method (Šimandl et al., 2006) could be adapted to design an optimized grid. For brevity and simplicity of presentation, a grid of sample points designed using the a priori information about the system and expert knowledge is considered in this paper. The non-grid points of the hyper-state space are mapped to the grid points using an aggregation function.

The grid points over the space of the reduced belief states $\mathbf{b}_{k}$ are designed considering the individual elements of $\mathbf{b}_{k}$ to be probabilities with values between zero and one. The grid points over the space of the variable $\mathbf{x}_{k}$ must take into account the dynamics of the individual models, values of admissible inputs and properties of the state noise.

Let us assume that the discrete sets $\mathcal{S}_{i}^{\mathrm{g}}$ representing 
quantization levels in the $i$-th dimension of the hyper-state $\mathbf{s}_{k}$ were chosen. Then, the grid of sample points is represented by the set $\mathcal{S}^{\text {g }}$ defined by the Cartesian product

$$
\mathcal{S}^{\mathrm{g}} \triangleq \mathcal{S}_{1}^{\mathrm{g}} \times \mathcal{S}_{2}^{\mathrm{g}} \times \ldots \times \mathcal{S}_{n_{s}}^{\mathrm{g}}=\left\{\overline{\mathbf{s}}^{i}\right\}_{i=1}^{N_{\mathrm{g}}},
$$

where $\overline{\mathbf{s}}^{i} \in \mathbb{R}^{n_{s}}$ is a grid point and $N_{\mathrm{g}}=$ $\left|\mathcal{S}_{1}^{\mathrm{g}}\right|\left|\mathcal{S}_{2}^{\mathrm{g}}\right| \ldots\left|\mathcal{S}_{n_{s}}^{\mathrm{g}}\right|$ denotes the total number of the grid points. As the total number of the grid points grows exponentially with the dimension of the hyper-state space, this approach is suited only to problems with a low dimension of the hyper-state. Note that, due to the way the set $\mathcal{S}^{\mathrm{g}}$ is created, it might contain grid points that are not consistent with the model. These grid points are simply left out from the further computation.

Assuming that the values of a function at the grid points are known, the value of the function at a non-grid point is approximated using an aggregation function $\mathrm{g}$ : $\mathcal{S} \mapsto \mathcal{S}^{\text {g }}$ that maps any hyper-state $\mathrm{s}_{k} \in \mathcal{S}$ to the "nearest" grid point $\overline{\mathbf{s}}_{k} \in \mathcal{S}^{\mathrm{g}}$. The aggregation function is assumed to be

$$
\overline{\mathbf{s}}_{k}=\mathbf{g}\left(\mathbf{s}_{k}\right)=\arg \min _{\boldsymbol{\xi} \in \mathcal{S}^{\mathrm{g}}}\left\|\mathbf{s}_{k}-\boldsymbol{\xi}\right\|_{2},
$$

where $\|\mathbf{z}\|_{2}$ is the $\mathrm{L}^{2}$ norm of a vector $\mathbf{z} \in \mathbb{R}^{n_{z}}$. Note that such an aggregation function defines a partition of the hyper-state space and the original function is approximated by a piecewise constant function.

3.3.2. Value iteration algorithm over grid points. The value iteration algorithm (Buşoniu et al., 2010) is used to compute successive approximations $\bar{V}^{(i)}$ of the optimal value function $\bar{V}^{*}$ over the uniform grid using the recursive equation

$$
\begin{array}{r}
\bar{V}^{(i+1)}(\boldsymbol{\xi}) \\
=\min _{d \in \mathcal{M}} \bar{L}^{\mathrm{d}}(\boldsymbol{\xi}, d)+\lambda \min _{\mathbf{u} \in \mathcal{U}} \mathrm{E}\left\{\bar{V}^{(i)}\left(\boldsymbol{\xi}^{\prime}\right) \mid \boldsymbol{\xi}, \mathbf{u}\right\}, \\
i=0,1, \ldots,
\end{array}
$$

where $\bar{V}^{(i)}: \mathcal{S}^{\mathrm{g}} \mapsto \mathbb{R}$ is an approximation of the optimal value function $\bar{V}^{*}$ at the $i$-th iteration, $\boldsymbol{\xi} \in \mathcal{S}^{\mathrm{g}}$ represents a grid point, and $\xi^{\prime} \in \mathcal{S}^{\text {q }}$ is a discrete random variable that represents an aggregated state resulting from using the input $\mathbf{u}$ while being at the grid point $\boldsymbol{\xi}$. The random variable $\xi^{\prime}$ is defined as

$$
\boldsymbol{\xi}^{\prime}=\mathbf{g}\left(\phi\left(\boldsymbol{\xi}, \mathbf{u}, \mathbf{x}^{\prime}\right)\right),
$$

where $\mathbf{x}^{\prime} \in \mathbb{R}^{n_{x}}$ corresponds to $\mathbf{x}_{k+1}$ regarded as an external disturbance. The initial condition for the value iteration algorithm is $\bar{V}^{(0)}(\boldsymbol{\xi})=0$ for all $\boldsymbol{\xi} \in \mathcal{S}^{\mathrm{g}}$. For each approximation $\bar{V}^{(i)}$, there is a corresponding suboptimal input signal generator $\bar{\gamma}^{(i)}$ defined as

$$
\mathbf{u}^{\mathrm{a}}=\overline{\boldsymbol{\gamma}}^{(i)}(\boldsymbol{\xi})=\arg \min _{\mathbf{u} \in \mathcal{U}} \mathrm{E}\left\{\bar{V}^{(i)}\left(\boldsymbol{\xi}^{\prime}\right) \mid \boldsymbol{\xi}, \mathbf{u}\right\} .
$$

Note that the time index $k$ is omitted since the particular time step does not play any role in the value iteration algorithm.

Since the convergence of the value iteration algorithm is discussed in literature (see, e.g., Buşoniu et al., 2010; Powell, 2007), only some important points are highlighted here. The sequence of the functions $\bar{V}^{(i)}$ asymptotically converges to the optimal value function $\bar{V}^{*}$ as the number of iteration $i$ goes to infinity. Since the iteration process is always terminated after a finite number of iterations $N_{\mathrm{vi}}$, there is a discrepancy between the optimal value function $\bar{V}^{*}$ and the value function $\bar{V} \bar{\gamma}$ that corresponds to the suboptimal input signal generator $\bar{\gamma}=$ $\bar{\gamma}^{\left(N_{\mathrm{vi}}\right)}$. Either a conservative estimate of the number of iterations required to attain a desired accuracy or the difference between two consecutive iterations is usually used as a stopping criterion. The value iteration algorithm is terminated at the iteration $i=N_{\mathrm{vi}}$ for which

$$
\left\|\bar{V}^{\left(N_{\mathrm{vi}}+1\right)}(\boldsymbol{\xi})-\bar{V}^{\left(N_{\mathrm{vi}}\right)}(\boldsymbol{\xi})\right\|_{\infty} \leq \delta,
$$

where $\|h(\mathbf{z})\|_{\infty}=\max _{\mathbf{z} \in \mathcal{Z}}|h(\mathbf{z})|$ is the maximum absolute value of a function $h$ over a countable set $\mathcal{Z}$ and $\delta>0$ is a chosen threshold. If a desired accuracy $\epsilon_{\mathrm{vi}}$ is given and it is required that $\left\|\bar{V}^{*}-\bar{V}^{\bar{\gamma}}\right\|_{\infty} \leq \epsilon_{\mathrm{vi}}$, the threshold $\delta$ can be computed as $\delta=\epsilon_{\mathrm{vi}}(1-\lambda) /(2 \lambda)$.

\subsubsection{Approximate computation of the conditional} expectation. The functional recursive equation (31) involves the computation of the conditional expected value

$$
\mathrm{E}\left\{\bar{V}^{(i)}\left(\mathbf{g}\left(\boldsymbol{\phi}\left(\boldsymbol{\xi}, \mathbf{u}, \mathbf{x}^{\prime}\right)\right)\right) \mid \boldsymbol{\xi}, \mathbf{u}\right\}
$$

It follows from the definition of the hyper-state and 18 that the conditional pdf

$$
p\left(\mathbf{x}^{\prime} \mid \boldsymbol{\xi}, \mathbf{u}\right)=p\left(\mathbf{x}_{k+1} \mid \mathbf{s}_{k}, \mathbf{u}_{k}\right)=p\left(\mathbf{x}_{k+1} \mid \mathbf{x}_{0}^{k}, \mathbf{u}_{0}^{k}\right)
$$

required to evaluate the expected value is a weighted sum of the predictive state pdfs conditioned by the models. As the aggregation function $\mathrm{g}$ and the approximate value functions $\bar{V}^{(i)}$ are non-linear, the expected value cannot be computed exactly. In the area of non-linear state estimation, the unscented transform (UT) has proven to be of much use when computing the statistics of a random variable that undergoes a non-linear transformation (Julier and Uhlmann, 1997). Using the UT, the expected value can be approximated as

$$
\begin{aligned}
\mathrm{E} & \left\{\bar{V}^{(i)}\left(\mathbf{g}\left(\boldsymbol{\phi}\left(\boldsymbol{\xi}, \mathbf{u}, \mathbf{x}^{\prime}\right)\right)\right) \mid \boldsymbol{\xi}, \mathbf{u}\right\} \\
& \approx \sum_{r=1}^{N} \sum_{q=0}^{2 n_{x}} w_{q} m_{r, q}^{\mathrm{t}} P\left(\mu_{k}=r \mid \mathbf{x}_{0}^{k}, \mathbf{u}_{0}^{k-1}\right),
\end{aligned}
$$


where $m_{r, q}^{\mathrm{t}}$ are transformed sigma points defined as

$$
m_{r, q}^{\mathrm{t}}=\bar{V}^{(i)}\left(\mathbf{g}\left(\boldsymbol{\phi}\left(\boldsymbol{\xi}, \mathbf{u}, \mathbf{m}_{r, q}\right)\right)\right) .
$$

The weights $w_{q}$ and the sigma points $\mathbf{m}_{r, q}$ are specified as

$$
\begin{aligned}
& w_{0}=\frac{\kappa}{n_{x}+\kappa}, \quad w_{s}=\frac{1}{2\left(n_{x}+\kappa\right)}, \quad s=1, \ldots, 2 n_{x}, \\
& \mathbf{m}_{r, 0}= \mathbf{f}_{r}(\mathbf{x}, \mathbf{u})+\mathbf{m}_{\mathbf{w}}(\mathbf{x}, r), \\
& \mathbf{m}_{r, q}=\mathbf{m}_{r, 0}-\left(\sqrt{\left(n_{x}+\kappa\right) \mathbf{P}_{\mathbf{w}}(\mathbf{x}, r)}\right)_{q}, \\
& q=1, \ldots, n_{x} \\
& \mathbf{m}_{r, q}= \mathbf{m}_{r, 0}+\left(\sqrt{\left(n_{x}+\kappa\right) \mathbf{P}_{\mathbf{w}}(\mathbf{x}, r)}\right)_{q-n_{x}}, \\
& q=n_{x}+1, \ldots, 2 n_{x}
\end{aligned}
$$

where $(\mathbf{M})_{i}$ denotes the $i$-th column of the matrix $\mathbf{M}, \mathbf{x}$ contains the first $n_{x}$ elements of the grid point $\boldsymbol{\xi}$ and $\kappa \in$ $\mathbb{R}$ is a design parameter.

3.3.4. Summary of approximate active fault detector design. This subsection summarizes the design of the approximate active fault detector. The detector is given by (27). The approximate Bellman function is computed off-line using Algorithm 1, which combines the results presented in the previous subsections. The approximate input signal generator is given by (28), where the exact Bellman function is replaced by its approximation.

$\overline{\text { Algorithm 1. Value iteration algorithm for finding the }}$ approximate Bellman function.

Step 1. Choose the set $\mathcal{S}^{\mathrm{g}}$ of grid points, initialize the approximate value function $\bar{V}^{(0)}(\boldsymbol{\xi})=0$ for all grid points $\boldsymbol{\xi} \in \mathcal{S}^{\mathrm{g}}$, choose the threshold $\delta$, and set $i=0$.

Step 2. Compute a new approximate value function $\bar{V}^{(i+1)}(\boldsymbol{\xi})$ for all $\boldsymbol{\xi} \in \mathcal{S}^{\mathrm{g}}$ using the functional recursive equation (31), approximation to the conditional mean value (37), and the aggregation function (30).

Step 3. If the termination condition (34) is not satisfied, set $i \leftarrow i+1$ and return to Step 2 .

\section{Numerical example}

The numerical example demonstrates the design of the approximate active fault detector for a second-order non-linear model of a pendulum with an intermittent fault that indicates development of wear in rolling bearings. The performance of the designed approximate active fault detector is evaluated and compared with other detection schemes where the same detector is used but different input signal generators are employed.
A non-linear state-space continuous-time model of a pendulum (Garces et al., 2003) is

$$
\left[\begin{array}{c}
\dot{x}_{1}(t) \\
\dot{x}_{2}(t)
\end{array}\right]=\left[\begin{array}{c}
x_{2}(t) \\
-\frac{g}{l} \sin \left(x_{1}(t)\right)-\frac{\beta}{m l^{2}} x_{2}(t)+\frac{1}{m l^{2}} u(t)
\end{array}\right],
$$

where $x_{1}(t)$ [rad] is the angular displacement from the vertically downward equilibrium position, $x_{2}(t)\left[\mathrm{rad} \mathrm{s}^{-1}\right]$ is the angular velocity, $u(t)[\mathrm{Nm}]$ is the input applied torque, $\beta\left[\mathrm{kg} \mathrm{m}^{2} \mathrm{~s}^{-1}\right]$ is the viscous damping coefficient, $m[\mathrm{~kg}]$ is the mass of the pendulum, $l[\mathrm{~m}]$ is the length of the pendulum, and $g \doteq 9.81\left[\mathrm{~m} \mathrm{~s}^{-2}\right]$ is the gravitational acceleration constant. The values of the physical parameters for the fault-free situation (Fault-free) and an intermittent fault (Fault) represented by a change in the viscous friction due to swarf or grit in the ball bearings are given in Table 1

The number of discrete-time non-linear models in the structure (2) is $N=2$. They are obtained using the forward Euler method with a sampling period of $T_{\mathrm{s}}=$ $0.05[\mathrm{~s}]$ in the form

$$
\mathbf{f}_{i}\left(\mathbf{x}_{k}, u_{k}\right)=\mathbf{A}_{i} \mathbf{x}_{k}+\mathbf{B} u_{k}+\mathbf{B}^{\mathrm{n}} \sin \left(x_{k, 1}\right),
$$

where $i \in \mathcal{M}=\{1,2\}$ and the matrices $\mathbf{A}_{i}, \mathbf{B}$ and $\mathbf{B}^{\mathrm{n}}$ are

$$
\begin{array}{ll}
\mathbf{A}_{1}=\left[\begin{array}{ll}
1 & 0.05 \\
0 & 0.85
\end{array}\right], & \mathbf{A}_{2}=\left[\begin{array}{cc}
1 & 0.05 \\
0 & 0.845
\end{array}\right], \\
\mathbf{B}=\left[\begin{array}{c}
0 \\
0.025
\end{array}\right], & \mathbf{B}^{\mathrm{n}}=\left[\begin{array}{cc}
0 \\
-0.4905
\end{array}\right] .
\end{array}
$$

The transition probabilities are assumed to be

$$
P\left(\mu_{k+1}=j \mid \mu_{k}=i\right)=\left[\begin{array}{ll}
0.98 & 0.02 \\
0.02 & 0.98
\end{array}\right]
$$

The set of possible input torques is $\mathcal{U}=$ $\{-10,0,10\}$. The components of the state noise vector $\mathbf{w}_{k}$ are assumed to be mutually independent and have the Laplace distribution (Forbes et al., 2011) with the location parameter $\eta=0$ and the scale parameter $\nu=$ $5.6569 \cdot 10^{-4}$. Thus, the mean value and the covariance matrix of the state noise are $\mathbf{m}_{\mathbf{w}}\left(\mathbf{x}_{k}^{\mathrm{a}}\right)=[\eta, \eta]^{\mathrm{T}}=[0,0]^{\mathrm{T}}$ and $\mathbf{P}_{\mathbf{w}}\left(\mathbf{x}_{k}^{\mathrm{a}}\right)=2 \nu^{2} \mathbf{I}_{2}=6.4 \cdot 10^{-7} \mathbf{I}_{2}$, respectively. The pdf of $\mathbf{x}_{0}$ is Gaussian with the zero mean value and the covariance matrix $0.0001 \mathbf{I}_{2}$. The system starts as fault free, i.e., $P\left(\mu_{0}=1\right)=1$. The discount factor is chosen to

Table 1. Values of the physical parameters of the pendulum.

\begin{tabular}{|l|c|c|}
\hline & Fault-free & Fault \\
\hline \hline$l[\mathrm{~m}]$ & 1 & 1 \\
$\beta\left[\mathrm{kg} \mathrm{m}^{2} \mathrm{~s}^{-1}\right]$ & 6 & 6.2 \\
$m[\mathrm{~kg}]$ & 2 & 2 \\
\hline
\end{tabular}


be $\lambda=0.98$ and the detection cost function is the zero-one cost function

$$
L^{\mathrm{d}}\left(\mu_{k}, d_{k}\right)= \begin{cases}0 & \text { if } d_{k}=\mu_{k} \\ 1 & \text { otherwise }\end{cases}
$$

When the problem is reformulated, the hyper-state has three components $\mathbf{s}_{k}=\left[x_{k, 1}, x_{k, 2}, b_{k, 1}\right]^{\mathrm{T}}$. Using (22), the equivalent detection cost function $\bar{L}^{\mathrm{d}}$ can be expressed as

$$
\begin{aligned}
\bar{L}^{\mathrm{d}}\left(\mathbf{s}_{k}, d_{k}\right) & =\sum_{\substack{i=1 \\
i \neq d}}^{N} P\left(\mu_{k}=i \mid \mathbf{x}_{0}^{k}, \mathbf{u}_{0}^{k-1}\right) \\
& = \begin{cases}1-s_{k, 3} & \text { if } d_{k}=1, \\
s_{k, 3} & \text { if } d_{k}=2 .\end{cases}
\end{aligned}
$$

It can easily be derived that the optimal fault detector is

$$
\begin{aligned}
d_{k}^{*}=\sigma^{*}\left(\mathbf{s}_{k}\right) & =\arg \min _{d_{k} \in \mathcal{M}} \bar{L}^{\mathrm{d}}\left(\mathbf{s}_{k}, d_{k}\right) \\
& = \begin{cases}1 & \text { if } s_{k, 3} \geq 0.5 \\
2 & \text { otherwise }\end{cases}
\end{aligned}
$$

and the corresponding minimum value of the detection cost is

$$
\min _{d_{k} \in \mathcal{M}} \bar{L}^{\mathrm{d}}\left(\mathbf{s}_{k}, d_{k}\right)=\min \left[1-s_{k, 3}, s_{k, 3}\right] .
$$

To design the approximate input signal generator, the Bellman functional equation is solved approximately over a non-uniform grid which is designed in such a way that its extent and quantization levels reflect the dynamics of the models, admissible values of the input, and state noise characteristics. As a result, the value iteration algorithm represented by the functional recursive equation (31) is performed over 228563 grid points in the hyper-state space at each iteration. Within the value iteration algorithm, the mean value of the approximate value function is computed using the UT with parameter $\kappa=3$. The convergence rate of the value iteration algorithm is depicted in Fig. 2. The threshold $\delta=0.01$ for terminating the value iteration algorithm is attained after $N_{\mathrm{vi}}=41$ iterations.

The approximate value function cannot be visualized directly. To provide a basic insight, a slice through the obtained approximate value function $\bar{V}^{(41)}$ as a function of the angular displacement $s_{k, 1}$ and angular velocity $s_{k, 2}$ for the fixed probability of the fault-free model $s_{k, 3}=0.9$ is shown in Fig. 3. The corresponding approximate function $\bar{\gamma}^{(41)}$ is given in Fig. 4 Although slightly different slices are obtained for other values of $s_{k, 3}$, they all show that the quality of detection heavily depends on the angular velocity. When the angular velocity approaches zero, the approximate value function rapidly increases (i.e., the quality of detection decreases) regardless of the angular displacement. It corresponds to common sense that the change in the viscous friction is detected more quickly and reliably when the pendulum is already moving than when it needs to be pushed to move.

The discussion in the previous paragraph might suggest that in this particular example a simple ad hoc strategy that constantly swings the pendulum could be applied to obtain input-output data that are informative enough for the detector to make correct decisions. To examine this question experimentally, the designed approximate active fault detector is compared with a passive one and three other active fault detectors that all share the same fault detector but use different input signal generators. The input signal generators considered are the following:

- zero input signal generator (passive detector),

$$
u_{k}=0
$$

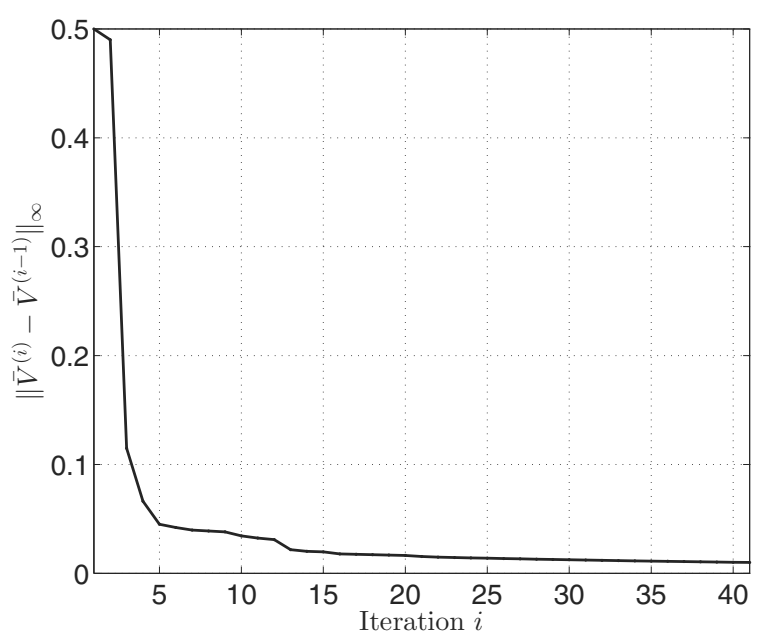

Fig. 2. Convergence rate of the value iteration algorithm.

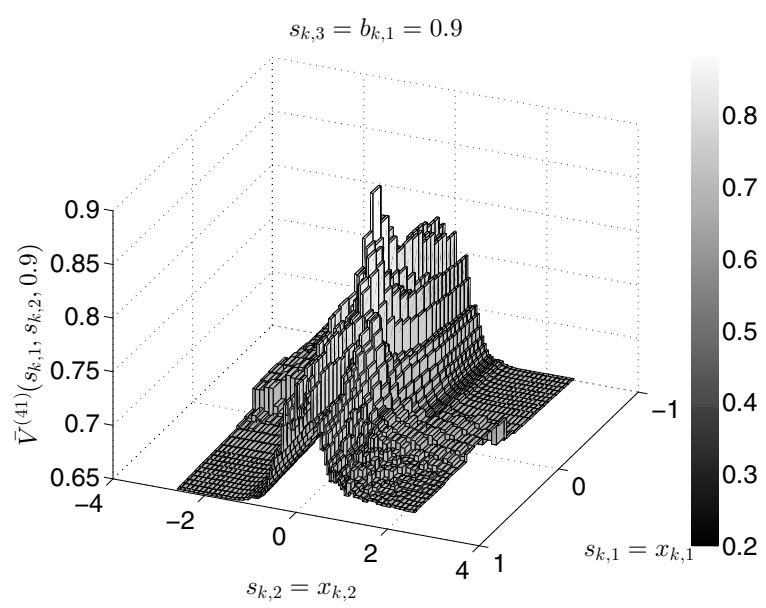

Fig. 3. Approximate value function $\bar{V}^{(41)}$ for $s_{k, 3}=0.9$. 
- random input signal generator,

$$
\begin{aligned}
P\left(u_{k}=-10\right) & =P\left(u_{k}=0\right) \\
& =P\left(u_{k}=10\right)=1 / 3,
\end{aligned}
$$

- sine input signal generator,

$$
u_{k}=\arg \min _{\bar{u} \in \mathcal{U}}|\bar{u}-10 \sin (0.05 \pi k)|
$$

- velocity switching input signal generator,

$$
u_{k}= \begin{cases}10 & \text { if } x_{k, 2} \geq 0 \\ -10 & \text { if } x_{k, 2}<0\end{cases}
$$

The detection quality of all fault detectors is evaluated on the finite horizon of $F_{\mathrm{FH}}=500$ steps, which equals $25[\mathrm{~s}]$. Note that the error caused by using only the finite horizon is bounded from above as

$$
\begin{array}{r}
\lim _{F \rightarrow \infty} \mathrm{E}\left\{\sum_{k=F_{\mathrm{FH}}}^{F} \lambda^{k} L^{\mathrm{d}}\left(\mu_{k}, d_{k}\right)\right\} \\
\leq \frac{\lambda^{F_{\mathrm{FH}}+1}}{1-\lambda} L_{\max }^{\mathrm{d}}=0.02 .
\end{array}
$$

Typical trajectories of the employed torque $u_{k}$, angular displacement $x_{k, 1}$, angular velocity $x_{k, 2}$, model index $\mu_{k}$ and computed probability of the fault-free model $P\left(\mu_{k}=1 \mid \mathbf{x}_{0}^{k}, u_{0}^{k-1}\right)$ for individual active fault detectors are shown in Figs. 54 14 Note that the probability of the fault-free model is depicted in the figures instead of the decisions because the probability provides the information about the decision itself and also the credibility of that decision. In the case of the zero input signal generator, it can be seen in Fig. 5

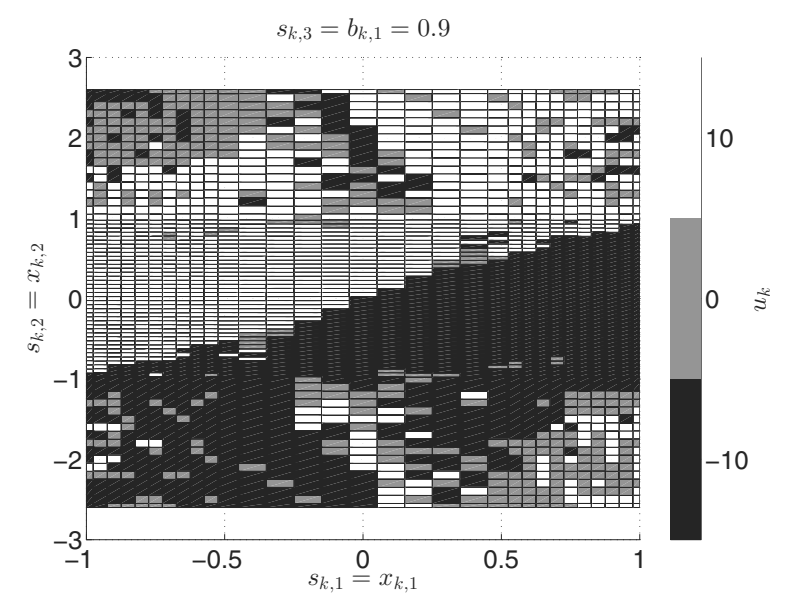

Fig. 4. Approximate input signal generator $\bar{\gamma}^{(41)}$ for $s_{k, 3}=0.9$ (light grey areas: $u_{k}=10$, grey areas: $u_{k}=0$, black areas: $\left.u_{k}=-10\right)$. that the excitation of the system provided by the state noise is insufficient and the probability of the fault-free model shown in Fig. 6 evolves mainly according to the a-priori information given by the transition probabilities. The random input signal generator provides a better input signal, but it does not drive either the angular displacement nor the angular velocity too far away from zero as indicated in Fig. 7 It consequently causes unreliable decisions being made, as can be observed in Fig. 8. It follows from Figs. 9 and 11 that the sine input signal generator and the velocity switching input signal generator provide a similar excitation to the system. Figures 10 and 12 suggest that more reliable decisions can be expected in comparison with the random input signal generator. The similarity of those two input signal generators is caused by the chosen period of the sine signal. Finally, Fig. 13 shows that the designed approximate closed loop input signal generator does not drive the angular displacement and angular velocity away from zero to such an extent as the previous two input signal generators do. Nevertheless, Fig. 14 demonstrates even a better detection quality of the designed approximate input signal generator.
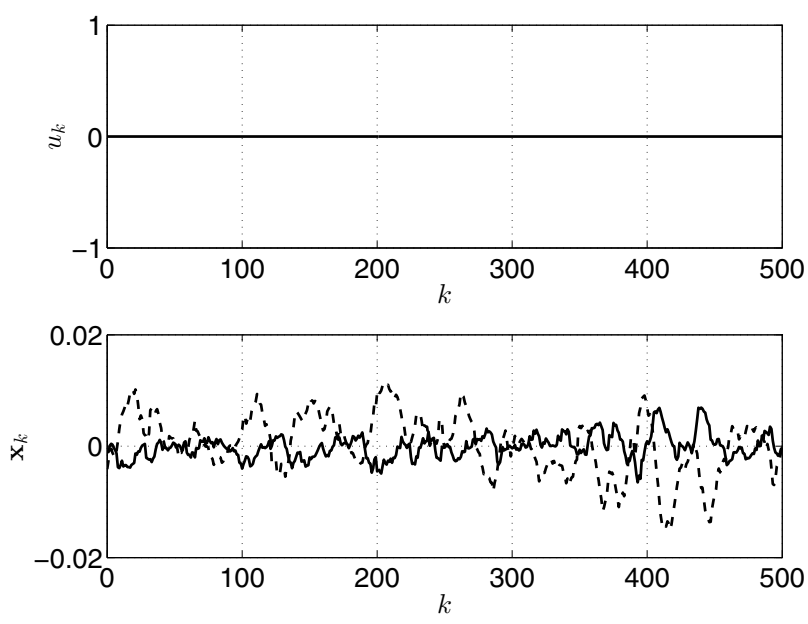

Fig. 5. Typical trajectories of the torque $u_{k}$ (top), angular displacement $x_{k, 1}$ (bottom-solid line), and angular velocity $x_{k, 2}$ (bottom-dotted line) for the zero input signal generator.

The presented typical trajectories illustrate the behaviour of the active fault detectors only for one particular realization of the state noise and switching between fault-free and faulty models. Although these typical trajectories indicate that, except for the zero input signal generator, the input signals allow the detector to generate sensible decisions, the detection quality across different random factors is evaluated using 10000 Monte Carlo simulations to obtain more convincing evidence. The estimated values of the criterion $J$ and the 

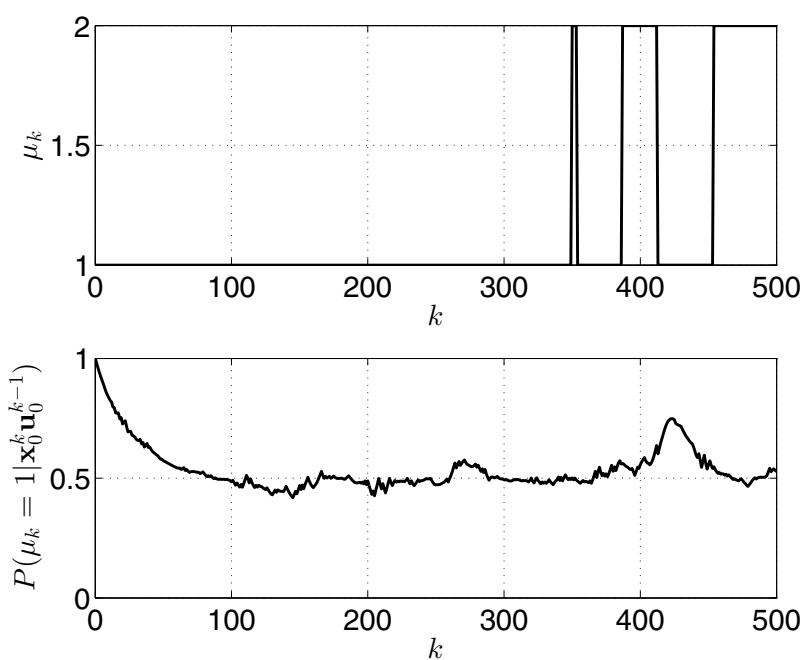

Fig. 6. Typical trajectories of the model $\mu_{k}$ (top) and probability of the fault-free model $P\left(\mu_{k}=1 \mid \mathbf{x}_{0}^{k}, u_{0}^{k-1}\right)$ (bottom) for the zero input signal generator.
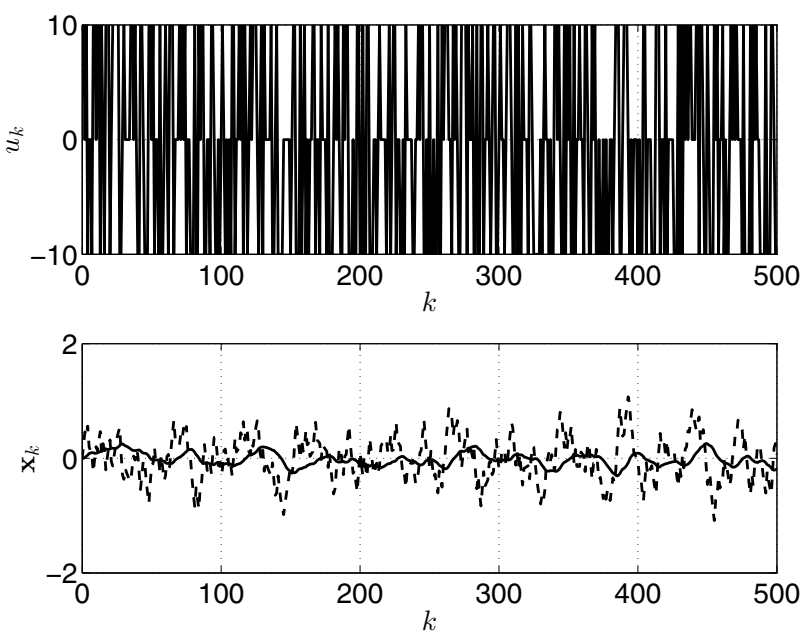

Fig. 7. Typical trajectories of the torque $u_{k}$ (top), angular displacement $x_{k, 1}$ (bottom-solid line), and angular velocity $x_{k, 2}$ (bottom-dotted line) for the random input signal generator.

precision of these estimates obtained using the bootstrap method (Efron and Tibshirani, 1994) are presented in Table 2 The results show that even if the error caused by evaluating the detection quality on a finite time horizon using Monte Carlo simulations is accounted for, the designed approximate active fault detector outperforms the other active fault detectors.
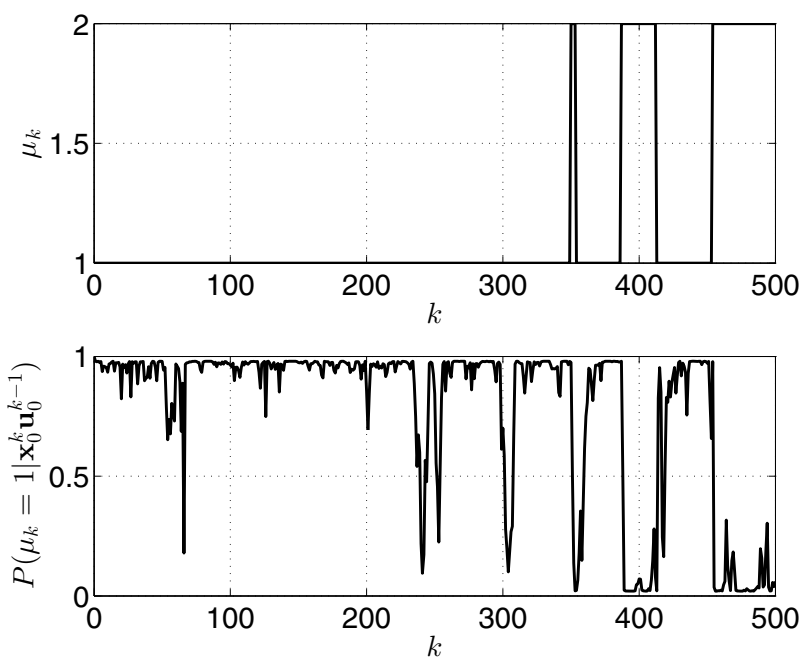

Fig. 8. Typical trajectories of the model $\mu_{k}$ (top) and probability of the fault-free model $P\left(\mu_{k}=1 \mid \mathbf{x}_{0}^{k}, u_{0}^{k-1}\right)$ (bottom) for the random input signal generator.
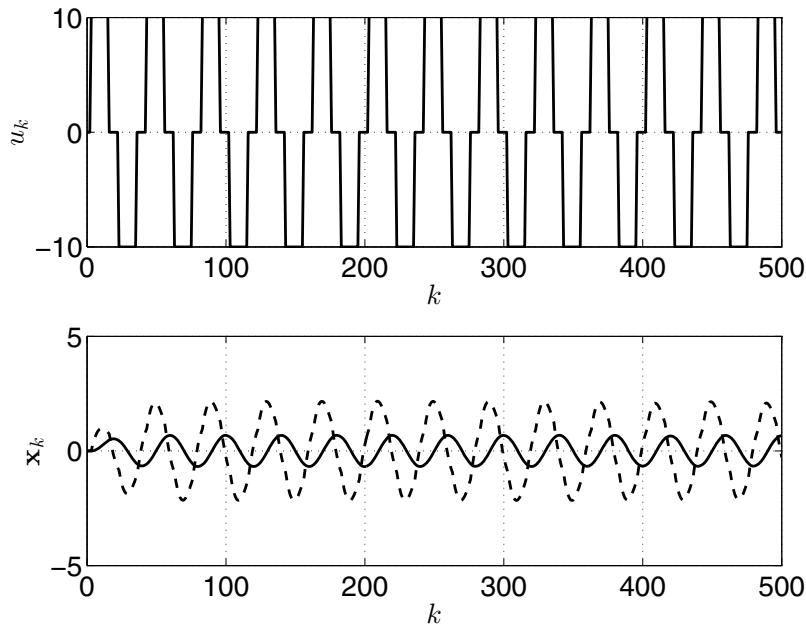

Fig. 9. Typical trajectories of the torque $u_{k}$ (top), angular displacement $x_{k, 1}$ (bottom-solid line), and angular velocity $x_{k, 2}$ (bottom-dotted line) for the sine input signal generator.

Table 2. Results of 10000 Monte Carlo simulations
\begin{tabular}{|l|c|c|}
\hline Input signal generator & $\hat{J}^{\mathrm{MC}}$ & $3 \delta_{\hat{J}^{\mathrm{MC}}}$ \\
\hline \hline Zero & 16.1919 & 0.3177 \\
Random & 3.0656 & 0.0673 \\
Sine & 1.4927 & 0.0398 \\
Velocity switching & 1.5708 & 0.0400 \\
Approximate closed loop & 1.2647 & 0.0287 \\
\hline
\end{tabular}



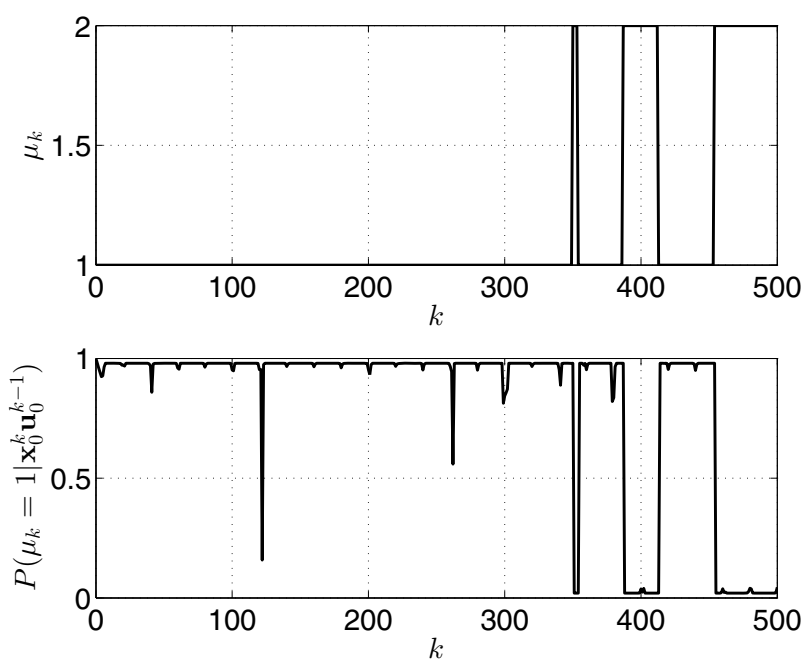

Fig. 10. Typical trajectories of the model $\mu_{k}$ (top) and probability of the fault-free model $P\left(\mu_{k}=1 \mid \mathbf{x}_{0}^{k}, u_{0}^{k-1}\right)$ (bottom) for the sine input signal generator.
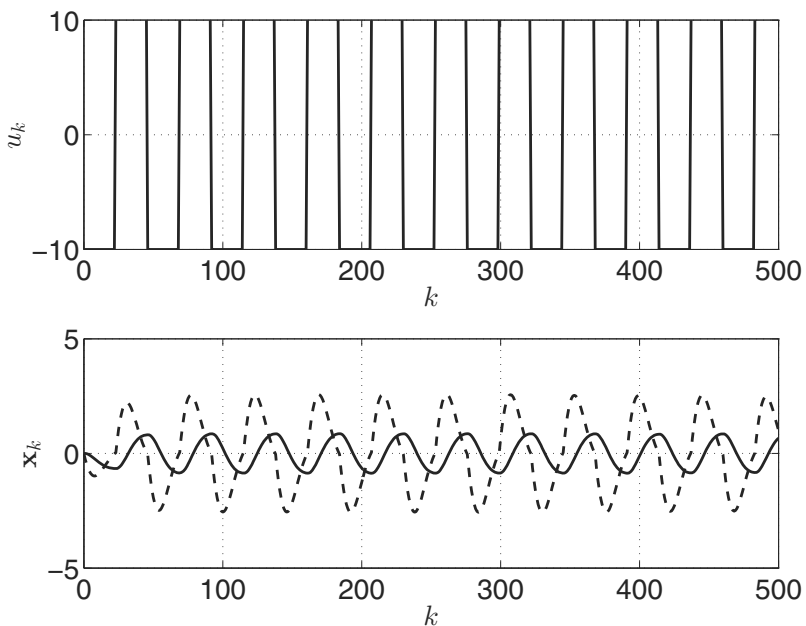

Fig. 11. Typical trajectories of the torque $u_{k}$ (top), angular displacement $x_{k, 1}$ (bottom-solid line), and angular velocity $x_{k, 2}$ (bottom-dotted line) for the velocity switching input signal generator.

\section{Conclusion}

The paper dealt with active fault diagnosis for a non-linear non-Gaussian system over an infinite time horizon. The design of an active fault detector was formulated as a discounted dynamic optimization problem with imperfect state information, reformulated as a perfect state information problem by introducing a new state called hyper-state, and solved by a technique of approximate dynamic programming. In comparison to finite time horizon problems, a simpler time-invariant
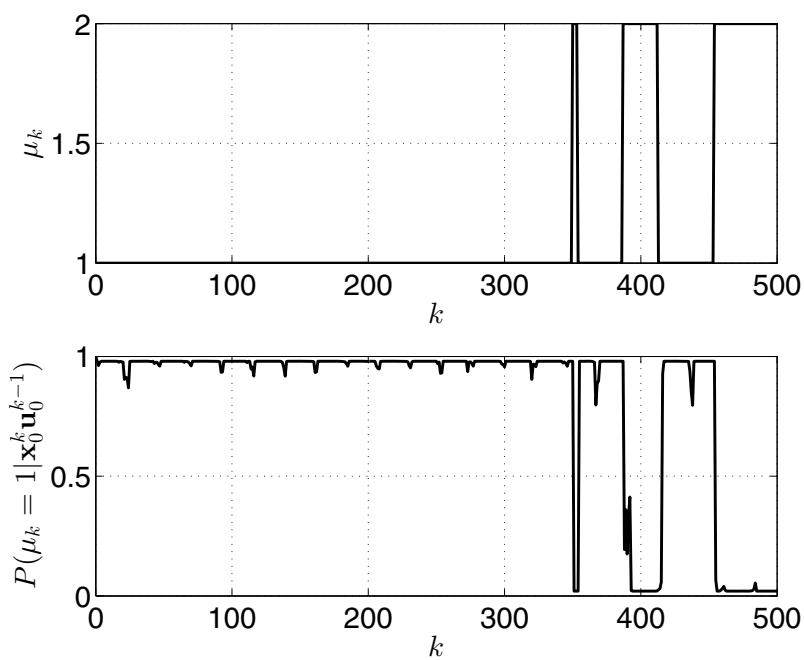

Fig. 12. Typical trajectories of the model $\mu_{k}$ (top) and probability of the fault-free model $P\left(\mu_{k}=1 \mid \mathbf{x}_{0}^{k}, u_{0}^{k-1}\right)$ (bottom) for the velocity switching input signal generator.
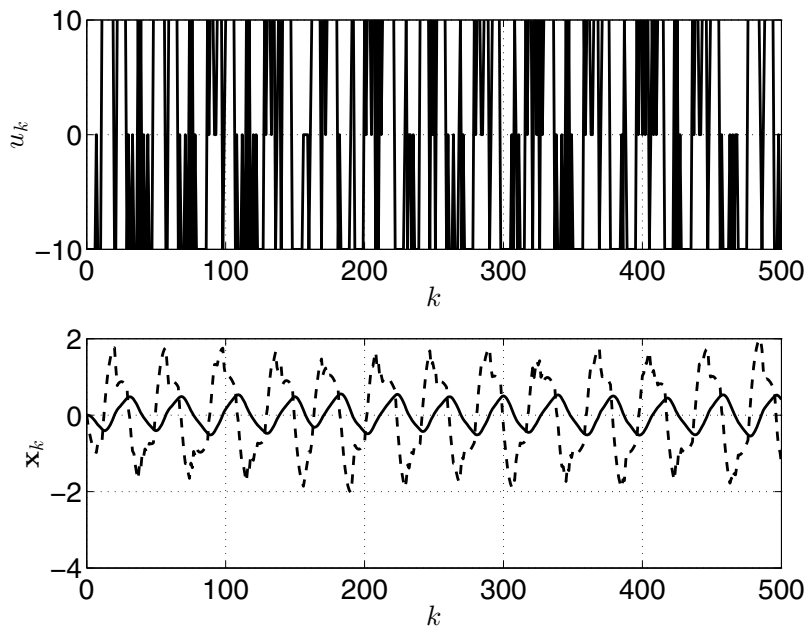

Fig. 13. Typical trajectories of the torque $u_{k}$ (top), angular displacement $x_{k, 1}$ (bottom-solid line), and angular velocity $x_{k, 2}$ (bottom-dotted line) for the designed approximate closed loop input signal generator.

solution is obtained in the case of infinite time horizon problems. The presented approach allows the optimal detector and corresponding approximate closed loop input signal generator to account for the stochastic nature of the system, including the unknown switching between fault-free and faulty conditions. As both the detector and the approximate input signal generator take the current value of the hyper-state into account while making the decision and generating the input, the quality of detection is increased compared to a passive detector or an active fault detector with an open loop input signal generator. 

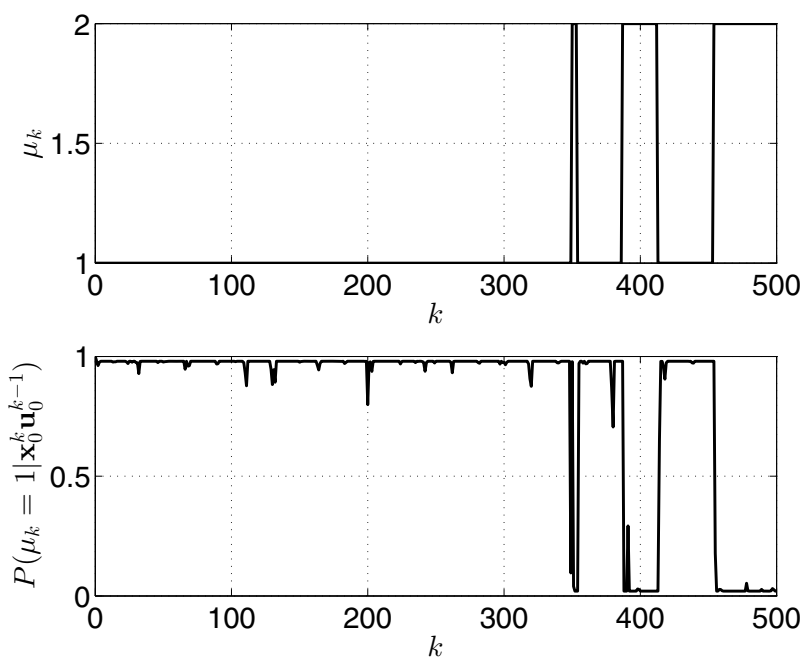

Fig. 14. Typical trajectories of the model $\mu_{k}$ (top) and probability of the fault-free model $P\left(\mu_{k}=1 \mid \mathbf{x}_{0}^{k}, u_{0}^{k-1}\right)$ (bottom) for the designed approximate closed loop input signal generator.

The presented approach can further be extended in several ways to allow a broader range of problems to be addressed. For example, it can be extended to allow for a continuous set of admissible inputs. Such an extension would require replacing the enumerative optimization at each iteration of the value iteration algorithm by a suitable derivative-free optimization algorithm. Another extension consists in taking potential constraints on the continuous part of the state into account. This kind of constraints could be systematically incorporated by making the set of admissible inputs depend on the state. The last extension worth mentioning is to allow a system where the continuous part of the state is partially observed through noisy measurements instead of being directly available. In this case, the conditional probability density function of the whole state can be used in the hyper-state as a sufficient statistic. However, this extension makes the problem more difficult because the dimension of the hyper-state is higher and a non-linear filtering technique has to be used to compute the required probability density function. All these extensions are beyond the scope of this paper and constitute interesting topics for future research.

\section{Acknowledgment}

This work was supported by the Czech Science Foundation (project no. GAP103/11/P407).

\section{References}

Andjelkovic, I., Sweetingham, K. and Campbell, S.L. (2008). Active fault detection in nonlinear systems using auxiliary signals, Proceedings of the 2008 American Control Conference, Seattle, WA, USA, pp. 2142-2147.

Ashari, A.E., Nikoukhah, R. and Campbell, S.L. (2012a). Active robust fault detection in closed-loop systems: Quadratic optimization approach, IEEE Transactions on Automatic Control 57(10): 2532-2544.

Ashari, A.E., Nikoukhah, R. and Campbell, S.L. (2012b). Effects of feedback on active fault detection, Automatica 48(5): 866-872.

Åström, K.J. (1965). Optimal control of Markov processes with incomplete state information, Journal of Mathematical Analysis and Applications 10(1): 174-205.

Atkinson, A.C. and Donev, A.N. (1992). Optimum Experimental Designs, Oxford University Press, New York, NY.

Bar-Shalom, Y. (1981). Stochastic dynamic programming: Caution and probing, IEEE Transactions on Automatic Control 26(5): 1184-1194.

Basseville, M. and Nikiforov, I.V. (1993). Detection of Abrupt Changes-Theory and Application, Prentice Hall, Englewood Cliffs, NJ.

Bertsekas, D.P. (1995). Dynamic Programming and Optimal Control, Volume I, Athena Scientific, Belmont, MA

Blackmore, L., Rajamanoharan, S. and Williams, B.C. (2008). Active estimation for jump Markov linear systems, IEEE Transactions on Automatic Control 53(10): 2223-2236.

Buşoniu, L., Babuška, R., Schutter, B.D. and Ernst, D. (2010). Reinforcement Learning and Dynamic Programming Using Function Approximators, CRC Press, Boca Raton, FL.

Campbell, S.L. and Nikoukhah, R. (2004). Auxiliary Signal Design for Failure Detection, Princeton University Press, Princeton, NJ.

Denardo, E.V. (2003). Dynamic Programming: Models and Applications, Dover Publications, Mineola, NY.

Efron, B. and Tibshirani, R.J. (1994). An Introduction to the Bootstrap, Chapman and Hall, New York, NY.

Forbes, C., Evans, M., Hastings, N. and Peacock, B. (2011). Statistical Distributions, 4th Edn., John Wiley \& Sons, Inc., Hoboken, NJ.

Garces, F., Becerra, V.M., Kambhampati, C. and Warwick, K. (2003). Strategies for Feedback Linearisation-A Dynamic Neural Network Approach, Springer-Verlag, London.

Goodwin, G.C. and Payne, R.L. (1977). Dynamic System Identification: Experiment Design and Data Analysis, Academic Press, New York, NY.

Isermann, R. (2006). Fault-Diagnosis Systems: An Introduction from Fault Detection to Fault Tolerance, Springer, Berlin.

Julier, S.J. and Uhlmann, J.K. (1997). New extension of the Kalman filter to nonlinear systems, Proceedings of the 1997 SPIE Conference on Signal Processing, Sensor Fusion, and Target Recognition, Orlando, FL, USA, pp. 182-193.

Kerestecioğlu, F. (1993). Change Detection and Input Design in Dynamical Systems, Research Studies Press, Taunton. 
Kiefer, J.C. (1959). Optimum experimental designs, Journal of the Royal Statistical Society (Series B) 21(2): 272-319.

Lee, J.M., Kaisare, N.S. and Lee, J.H. (2006). Choice of approximator and design of penalty function for an approximate dynamic programming based control approach, Journal of Process Control 16(2): 135-156.

Mehra, R.K. (1974). Optimal input signals for parameter estimation in dynamic systems-Survey and new results, IEEE Transactions on Automatic Control 19(6): 753-768.

Nett, C.N. (1986). Algebraic aspects of linear control system stability, IEEE Transactions on Automatic Control 31(10): 941-949.

Niemann, H.H. (2006). A setup for active fault diagnosis, IEEE Transactions on Automatic Control 51(9): 1572-1578.

Niemann, H.H. (2012). A model-based approach to fault-tolerant control, International Journal of Applied Mathematics and Computer Science 22(1): 67-86, DOI: 10.2478/v10006-012-0005-x.

Poulsen, N.K. and Niemann, H. (2008). Active fault diagnosis based on stochastic tests, International Journal of Applied Mathematics and Computer Science 18(4): 487-496, DOI: 10.2478/v10006-008-0043-6.

Powell, W.B. (2007). Approximate Dynamic Programming: Solving the Curses of Dimensionality, Wiley-Interscience, Hoboken, NJ.

Puig, V. (2010). Fault diagnosis and fault tolerant control using set-membership approaches: Application to real case studies, International Journal of Applied Mathematics and Computer Science 20(4): 619-635, DOI: 10.2478/v10006-010-0046-y.

Puterman, M.L. (2005). Markov Decision Processes-Discrete Stochastic Dynamic Programming, John Wiley \& Sons, Hoboken, NJ.

Scola, H.R., Nikoukhah, R. and Delebecque, F. (2003). Test signal design for failure detection: A linear programming approach, International Journal of Applied Mathematics and Computer Science 13(4): 515-526.

Scott, J.K., Findeisen, R., Braatz, R.D. and Raimondo, D.M. (2013). Design of active inputs for set-based fault diagnosis, Proceedings of the 2013 American Control Conference, Washington, DC, USA, pp. 3561-3566.

Šimandl, M., Královec, J. and Söderström, T. (2006). Advanced point-mass method for nonlinear state estimation, Automatica 42(7): 1133-1145.

Šimandl, M. and Punčochář, I. (2009). Active fault detection and control: Unified formulation and optimal design, Automatica 45(9): 2052-2059.
Šimandl, M., Punčochář, I. and Královec, J. (2005). Rolling horizon for active fault detection, Proceedings of the 44th IEEE Conference on Decision and Control/European Control Conference 2005, Seville, Spain, pp. 3789-3794.

Široký, J., Šimandl, M., Axehill, D. and Punčochář, I. (2011). An optimization approach to resolve the competing aims of active fault detection and control, Proceedings of the 50th IEEE Conference on Decision and Control/European Control Conference, Orlando, FL, USA, pp. 3712-3717.

Zhang, X.J. (1989). Auxiliary Signal Design in Fault Detection and Diagnosis, Springer-Verlag, Berlin.

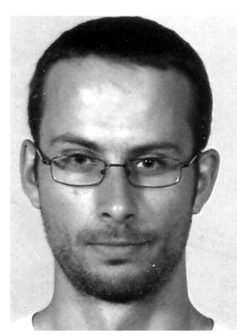

Ivo Punčochář received the M.Sc. (Ing.) degree in control engineering in 2003 and the Ph.D. degree in cybernetics in 2008, both from the University of West Bohemia, Pilsen, Czech Republic. He is currently a research scientist at the Department of Cybernetics, Faculty of Applied Sciences, University of West Bohemia. His research interests include active fault detection and optimal stochastic control.

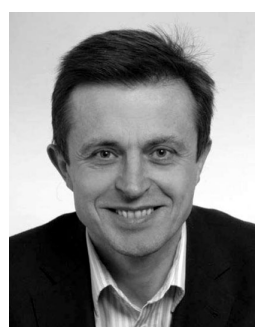

Miroslav Šimandl received his M.Sc. (Ing.) degree in control engineering in 1978 and his Ph.D. (CSc.) degree in technical cybernetics in 1984, both from the Institute of Technology in Pilsen, Czechoslovakia. Since 2002 he has held the position of a professor in the Department of Cybernetics, Faculty of Applied Sciences, University of West Bohemia, Pilsen, Czech Republic. Within the International Federation of Automatic Control (IFAC) he served as the co-chair of the IPC of the 16th IFAC World Congress in Prague (2005) and as a member of two technical committees of the IFAC. Professor Šimandl is the author or a co-author of many journal and conference technical papers (more than 140). His main research interests are in the fields of nonlinear filtering, active fault detection, nonlinear identification and adaptive dual control.

Received: 18 October 2013 Revised: 28 March 2014 